\title{
Evaluación genotóxica del agua del Río Grande (Antioquia, Colombia) mediante frecuencia de eritrocitos micronucleados de Brycon henni (Characiformes: Characidae)
}

\author{
Lina M. Zapata-Restrepo, Luz Y. Orozco-Jiménez, Maribel Rueda-Cardona, \\ Sandra L. Echavarría, Nehir Mena-Moreno \& Jaime A. Palacio-Baena \\ Grupo de Investigación en Gestión y Modelación Ambiental (GAIA)- Universidad de Antioquia - Sede de \\ Investigación Universitaria (SIU), Calle 62 No. 52-59, Medellín, Colombia; zapata.lina@gmail.com, \\ lyorozcoj@gmail.com,mariboo1379@gmail.com, lilitoo@gmail.com, nehirmena@gmail.com, \\ jpalaciob@gmail.com
}

\section{Recibido 11-XII-2015. Corregido 19-VII-2016. Aceptado 19-VIII-2016.}

\begin{abstract}
Genotoxic evaluation of Río Grande (Antioquia, Colombia) water using micronucleus frequency in erythrocytes of Brycon henni (Characiformes: Characidae). The permanent monoculture of bananas and plantains farming in the middle of Río Grande (Turbo - Antioquia) requires the application of a variety of pesticides. Inappropriate banana production practices in this region, have often led to waterbody pollution by agrochemicals from leachate and runoff processes. Currently, fish are the most common vertebrates used as bioindicators of water quality, because they are very sensitive to the presence of contaminants. Our main goal with this study was to compare the frequency of micronucleated erythrocytes (MNE) in peripheral blood specimens of fish Brycon henni, from two locations (polluted and unpolluted) in the Rio Grande. We evaluated the frequency of MNE in peripheral blood samples of fish $B$. henni from each location during two rainy seasons in 2010 and two dry seasons in 2011. Blood samples were collected, fixed for $24 \mathrm{~h}$, and then were stained with Giemsa. Among results, we found that the median frequency of MNE was higher in the polluted site by agrochemical discharges $(0.15 \pm 0.18)$, than in the unimpacted site $(0.06 \pm 0.08)$. Furthermore, the frequency of MNE in $B$. henni during the dry season was highly significant for both locations. The results of this study indicated that the analysis of MNE in B. henni could be recommended as a suitable method for in situ detection of environmental genotoxins. Rev. Biol. Trop. 65 (1): 405-414. Epub 2017 March 01.
\end{abstract}

Key words: frequency of micronucleated erythrocytes, biomarker, agrochemicals, Brycon henni, DNA damage.

El desarrollo agrícola ha conducido al incremento del uso de agroquímicos para el control de plagas en zonas de cultivos. Los efectos adversos observados para estas sustancias se atribuyen a su capacidad para atravesar las membranas biológicas e interactuar directamente o a través de sus metabolitos con blancos celulares (proteínas, lípidos y material genético) (Al Sabti, 1985; Al-Sabti \& Metcalfe, 1995; Goksøyr, 1991) produciendo cambios fisiológicos y con efectos adversos sobre el crecimiento, la reproducción, la longevidad y la sobrevivencia de los organismos (Anderson \& Wild, 1994; Shugart \& Theodorakis, 1998).
Aunque la evaluación de los efectos de contaminantes sobre la calidad de aguas de río son evaluados a través la detección y cuantificación de estos agentes mediante procedimientos de química analítica, hoy en día se recomienda incluir indicadores que permitan evaluar los efectos adversos sobre el ADN de la biota expuesta (Akpoilih, 2012; Osman, 2014). Los peces son excelentes bioindicadores ya que son muy sensibles a la presencia de contaminantes en el ambiente, y por esto son ampliamente utilizados en estudios de monitoreo ambiental como indicadores que permiten estimar los posibles efectos biológicos 
causados por una gran variedad de compuestos presentes en los ambientes acuáticos (Çavaş \& Ergene-Gozukara, 2005; De Flora, Bagnasco, \& Zanacchi, 1991; De Flora et al., 1993, Goksøyr, 1991; Lakra \& Nagpure, 2009, Obiakor, Okonkwo, Nnabude, \& Ezeonyejiaku, 2012; Udroiu, 2006).

La determinación de la frecuencia de eritrocitos micronucleados (EMN) es uno de los biomarcadores de efecto más empleados para evaluar los efectos genotóxicos y mutagénicos de los contaminantes presentes en el ambiente debido a que es un método sensible, rápido y se puede emplear fácilmente en condiciones de campo y de laboratorio (Al-Sabti \& Metcalfe, 1995; Ali, Nagpure, Kimar, Kumar, \& Kushwaha, 2008; Çavaş \& Ergene-Gözükara, 2005; Jha, 2004). Los micronúcleos (MN) se forman durante la división celular como resultado de ruptura cromosómica (clastogenicidad) y/o disfunción del huso mitótico (aneugenicidad) (Al-Sabti \& Metcalfe, 1995), por lo que su detección da cuenta de posibles efectos a causa de daño en el ADN. Este biomarcador ha sido ampliamente utilizado en peces porque permite la detección temprana de problemas ambientales en los ecosistemas acuáticos (Al-Sabti \& Metcalfe, 1995, Ergene et al., 2007; Grisolia \& Starling, 2001; Lopez-Barea, 1996; Polard et al., 2011b; Talapatra et al., 2007).

La cuenca media del Rio Grande se encuentra ubicada en Turbo, municipio localizado en la subregión de Urabá en el departamento de Antioquia (Colombia). Es un importante recurso para la población aledaña porque provee de zonas de pesca, agua de consumo y una fuente de riego para muchos cultivos en la región (Mintrabajo, 2013; Zamora \& García-Valencia, 2007).

Para el 2012 la zona agrícola en Turbo ocupaba una extensión aproximada de 28610 ha (Mintrabajo, 2013). Esta actividad se desarrolla en forma intensiva principalmente debido al cultivo de banano y plátano que constituyen el primer producto agrícola de la región (Mintrabajo, 2013; Zamora \& García-Valencia, 2007). Estos monocultivos demandan la aplicación aérea de agroquímicos, especialmente del herbicida glifosato y del insecticida clorpirifos, cuya presencia ha sido detectada en las fuentes hídricas de esta región (CORPOURABA, 2007; INVEMAR, 2004; Vivas-Aguas et al., 2011; Bonilla, Peinado, Urdaneta, \& Carrascal 2000) y sus efectos genotóxicos han sido reportados en una gran variedad de organismos (Çavaş \& Könen, 2007; Cavas, 2011; Giesy, Dobson, \& Solomon, 2000; Gollapudi, Mendrala, \& Linscombe, 1995; Murthy, Kiran, \& Venkateshwarlu, 2013; Nwani et al., 2010, 2011; Rahman, Mahboob, Danadevi, Banu, \& Grover, 2002; Ruiz \& Marzin, 1997; Ullah \& Zorriehzahra, 2015). Adicionalmente, la contaminación de aguas superficiales con estas sustancias se da como resultado de procesos de escurrimientos pluviales y lixiviación (Polard et al., 2011a), los cuales se ven favorecidos en esta zona por una continua precipitación.

Brycon henni (Eigenmann, 1913), de la subfamilia Bryconinae, comúnmente conocida como sabaleta, es una especie dulceacuícola endémica de Colombia, que habita agua dulce, que se encuentra ampliamente distribuido en la región occidental de Colombia (Montoya-López, Carrillo, \& Olivera-Ángel, 2006a). $B$. henni es una especie omnívora de alta adaptabilidad alimenticia, esquiva, gregaria y no migradora, y presenta dimorfismo sexual (Montoya-López et al., 2006a). Esta especie es importante como fuente de alimento para los pobladores ribereños de los cuerpos de agua que habita, además de ser el principal recurso pesquero tradicional en algunas zonas del país. Además, es una especie que ha demostrado sensibilidad ante la presencia de sustancias genotóxicas en el ambiente (Hurtado-Alarcón, Solarte-David, López-Ortiz, \& MontoyaHerrera, 2007).

El objetivo de esta investigación fue comparar la frecuencia de EMN en sangre periférica de ejemplares de peces sabaleta (Brycon henni, Characiformes: Characidae) procedentes de dos estaciones de muestreo del Río Grande en Turbo (Golfo de Urabá, Antioquia) localizados río arriba y río abajo de una zona de cultivos con descargas directas de agroquímicos durante dos épocas climáticas contrastantes. 


\section{MATERIALES Y MÉTODOS}

Área de estudio: La cuenca del Río Grande comprende un área de $150 \mathrm{~km}^{2}$ y una longitud aproximada de $27 \mathrm{Km}$ (Fig. 1). Nace en el alto Quimarí (670 msnm), Serranía de Abibe y vierte sus aguas al Río León (15 msnm), su caudal medio multianual es de $3.90 \mathrm{~m}^{3} / \mathrm{s}$. Está ubicado en el extremo suroriental del municipio de Turbo y al noroccidente del departamento de Antioquia (Colombia). En la zona, se presenta una época de lluvia de abril a noviembre, y una época seca de enero a marzo; la precipitación promedio anual fluctúa entre 2900 a $4100 \mathrm{~mm}$, la temperatura ambiente varía entre 26 a $28^{\circ} \mathrm{C}$, la humedad relativa registra valores promedios mensuales entre el 83 y $86 \%$, y predominan los vientos alisios del norte.

Estaciones de muestreo: La estación 1 se ubica en las coordenadas $7^{\circ} 56.45^{\prime} 4^{\prime \prime} \mathrm{N}$ - 76³6.14’00” W, en una zona en la que predominan bosques primarios y secundarios, ha sido poco alterada por actividades antrópicas, y no se presentan plantaciones de banano en el área. La estación 2, localizada en las coordenadas $7^{\circ} 56.23^{\prime} 4^{\prime} " \mathrm{~N}-76^{\circ} 36.42^{\prime} 2.0^{\prime \prime} \mathrm{W}$, se encuentra en un área afectada por las descargas
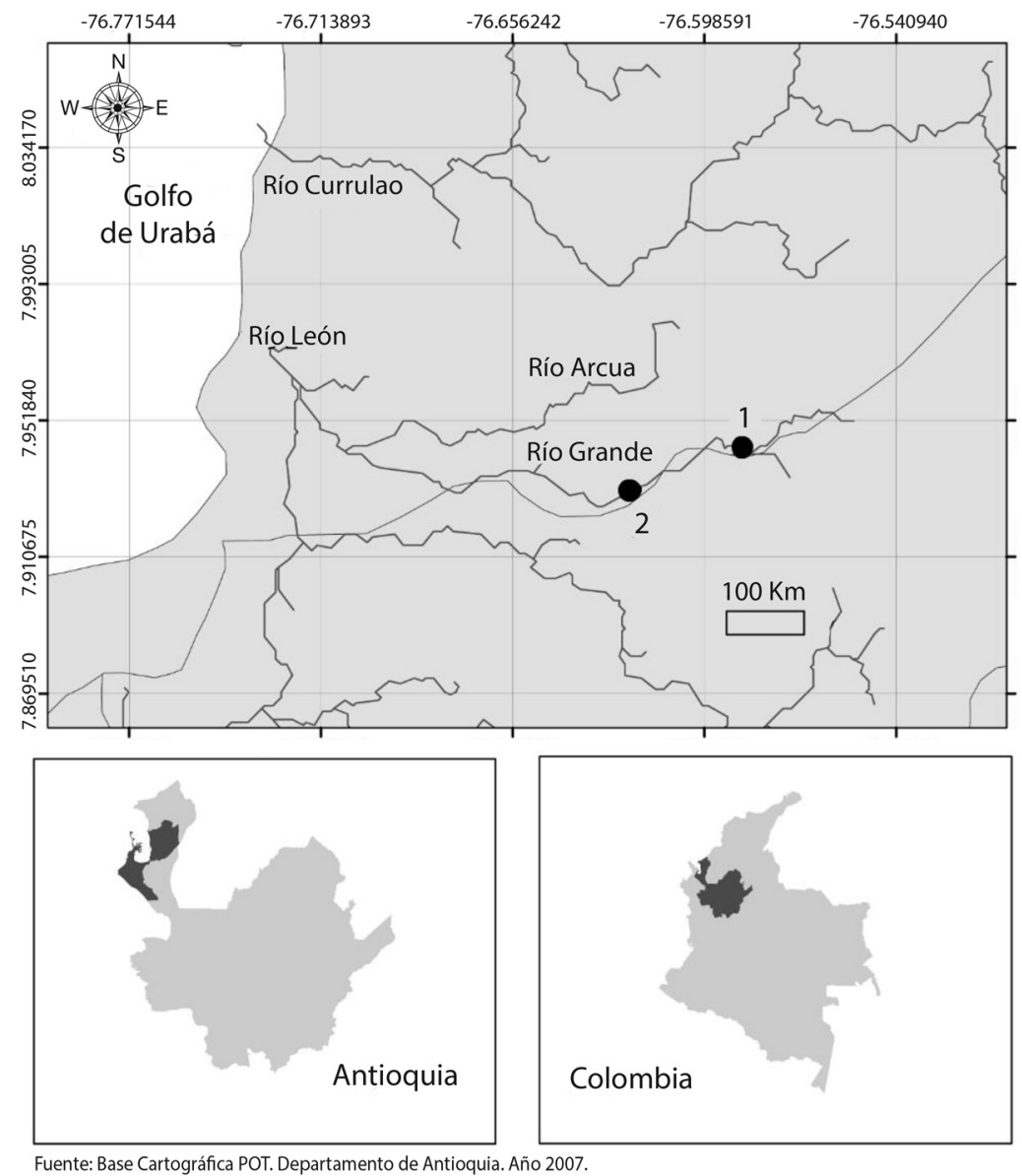

Información de referencia: Sistema de coordenadas geográficas: WGS 1984. Unidades: Grados decimales. Fecha: Agosto 2015.

Fig. 1. Área de estudio y estaciones de muestreo (círculos negros) en Río Grande (Turbo-Antioquia). 1 = zona poco alterada sin intervención antrópica; 2 = zona alterada por descargas de agroquímicos.

Fig. 1. Study area and sampling stations (black circles) in Rio Grande (Turbo-Antioquia). 1 = unimpacted area without human intervention; 2 = area polluted by discharges of agrochemicals. 
directas de agroquímicos procedentes de los cultivos de banano y plátano (Fig. 1).

Trabajo de campo: En cada estación se hicieron campañas de muestreo a lo largo de una época de lluvias (mayo a octubre) 2010 y una época seca (enero a marzo) 2011. En cada estación de muestreo se colectaron 18 ejemplares de $B$. henni durante la época de lluvias y 18 ejemplares en la época seca, para un total de 36 individuos por estación. En cada estación se tomaron medidas de los siguientes parámetros fisicoquímicos en el agua: temperatura $\left({ }^{\circ} \mathrm{C}\right), \mathrm{pH}$, oxígeno disuelto $(\mathrm{OD})(\mathrm{mg} / \mathrm{L})$, dióxido de carbono libre $\left(\mathrm{CO}_{2}\right)(\mathrm{mg} / \mathrm{L})$, porcentaje de saturación de oxígeno y alcalinidad (mg/L) (Cuadro 1).

La especie B. henni presenta una forma ovalada con coloración verdosa en el dorso y plateada en los costados, presenta una mancha en el opérculo y en la aleta caudal, además manchas rojizas en las aletas y mancha roja en la parte superior del ojo, es de tamaño pequeño a mediano, algunas veces crece hasta los 30 $\mathrm{cm}$ de longitud. $B$. henni es una especie que no aparece en ninguna de las categorías de riesgo propuestas por el libro rojo de peces dulceacuícolas de Colombia (Mojica, Castellanos, Usma, \& Álvarez, 2002) ni tampoco en la lista de especies amenazadas por la UICN del 2003, además por uno de los principales recursos pesqueros en algunas zonas se presenta como alternativa de seguridad alimentaria para las poblaciones rivereñas de los ríos en los que habita. El trabajo de campo fue realizado con apoyo de los pescadores de la zona, por lo que todos los organismos analizados fueron especímenes capturados con atarraya en sesiones de pesca rutinaria. Por las anteriores razones no se necesitaron permisos especiales para su captura.

Se seleccionaron para el estudio aquellos individuos que superaron $\operatorname{los} 10 \mathrm{~cm}$ de longitud, tamaño que indica que el pez es adulto (Montoya-López, Tabares, Echeverri, Arboleda, \& Olivera-Ángel, 2006b). A cada individuo le fue determinado el peso y longitud total (desde el extremo de la boca hasta el extremo de la aleta caudal con un ictiómetro convencional). El factor de condición por individuo se estimó mediante el índice de Fulton (K) (Ricker, 1975); $K=100\left(W / L^{3}\right)$, donde $W$ es el peso corporal húmedo en gramos y $L$ la longitud en $\mathrm{cm}$.

Los peces fueron sacrificados con una sobredosis de Eugenol (Rubio \& Silveira, 2009) e inmediatamente se obtuvo sangre periférica heparinizada $(0.5 \mathrm{~mL})$ de la vena caudal de cada individuo siguiendo la metodología propuesta por Nepomuceno y Spanó (1992) y Nepomuceno, Ferrari, Spanó y Centeno (1997). A partir de cada muestra se realizaron dos extendidos de sangre por individuo en portaobjetos limpios, los cuales se dejaron secar

CUADRO 1

Valores medios de los parámetros fisicoquímicos del agua para las estaciones de muestreo en el Río Grande y las dos épocas climáticas

TABLE 1

Means of physicochemical parameters of water found in the sampling stations in Río Grande and both seasons of studied period

\begin{tabular}{lcccc} 
& \multicolumn{2}{c}{ Estación 1 } & \multicolumn{2}{c}{ Estación 2} \\
& Época de lluvias & Época seca & Época de lluvias & Época seca \\
Temperatura $\left({ }^{\circ} \mathrm{C}\right)$ & $25.5 \pm 0.28$ & $27.8 \pm 0.49$ & $26.4 \pm 0.35$ & $28.0 \pm 0.56$ \\
$\mathrm{pH}$ & $7.77 \pm 0.08$ & $8.01 \pm 0.16$ & $7.64 \pm 0.18$ & $7.95 \pm 0.13$ \\
$\mathrm{OD}(\mathrm{mg} / \mathrm{L})$ & $7.77 \pm 0.26$ & $7.19 \pm 0.11$ & $7.54 \pm 0.16$ & $7.14 \pm 0.17$ \\
$\mathrm{CO}_{2}(\mathrm{mg} / \mathrm{L})$ & $1.47 \pm 0.11$ & $0.97 \pm 0.25$ & $1.98 \pm 0.74$ & $1.15 \pm 0.09$ \\
$\%$ Saturación de $\mathrm{O}_{2}$ & $94.74 \pm 2.78$ & $91.36 \pm 0.44$ & $93.31 \pm 1.44$ & $90.94 \pm 1.34$ \\
Alcalinidad $(\mathrm{mg} / \mathrm{L})$ & $42.5 \pm 3.54$ & $50.5 \pm 3.53$ & $44 \pm 4.2$ & $54 \pm 4.24$ \\
\hline
\end{tabular}


al aire durante 24 horas antes de ser procesados en el laboratorio. Los organismos fueron depositados en un recipiente conteniendo formalina al $10 \%$ y transportados al laboratorio de Hidrobiología de la Universidad de Antioquia, Seccional Urabá, donde fue confirmada taxonómicamente la especie usando la clave descrita por Dahl (1971).

Trabajo de laboratorio: En el laboratorio los frotis de sangre se fijaron con un mezcla de Metanol: Ácido Acético (3:1) durante siete minutos, se dejaron secar a temperatura ambiente y se tiñeron con Giemsa al $5 \%$ en tampón Sorensön pH 6.7 durante 20 minutos. Finalmente, las placas se lavaron con agua destilada y se dejaron secar a temperatura ambiente hasta su lectura y análisis.

Test de Micronúcleos: En cada lámina se contaron 1000 eritrocitos con el objetivo de 100x en un microscopio óptico (marca Olympus, modelo CH30) para un total de 2000 células por ejemplar. Para la identificación de los MN se siguieron los criterios propuestos por Fenech (2000) que indican que el tamaño debe ser entre 1/6 a 1/3 del diámetro del núcleo principal, deben estar claramente separados de la membrana nuclear y presentar igual coloración al núcleo principal. La frecuencia de EMN se determinó a partir de la siguiente expresión: Frecuencia de $\mathrm{EMN}=($ No. de células con micronúcleo x 1000)/No. total de células contadas.

Los datos se presentan como media \pm D.E. Para determinar su comportamiento paramétrico o no paramétrico se usaron las pruebas de Kolmogorov Smirnov y Shapiro Wilk. Mediante la prueba de Wilcoxon se analizó la homogeneidad de los muestreos por estación de muestreo. Las diferencias entre las estaciones y la incidencia de la época climática se determinaron mediante la prueba de Mann-Whitney. La relación entre los parámetros biométricos (longitud, peso y factor de condición) y la frecuencia de EMN fue determinada por medio de una correlación de Spearman. En todos los casos, las diferencias fueron consideras estadísticamente significativas si $\mathrm{P}<0.05$.

\section{RESULTADOS}

Todos los parámetros fisicoquímicos variaron las épocas climáticas, siendo mayor la temperatura, el $\mathrm{pH}$ y la alcalinidad del agua durante la época seca en las dos estaciones, mientras que los valores de oxígeno disuelto, dióxido de carbono y porcentaje de saturación del oxígeno fueron mayores durante la época de lluvias. No hubo diferencias entre los parámetros fisicoquímicos al comparar las estaciones de muestreo independientemente de la época climática (Cuadro 1).

Incidencia de factores biométricos en la frecuencia de EMN: Se encontró una relación significativa entre longitud-peso (Spearman, $\left.r_{\mathrm{s}}=0.91, \mathrm{P}=0.0000\right)$, longitud-factor de condición (Spearman, $\mathrm{r}_{\mathrm{s}}=0.32, \mathrm{P}=0.0064$ ) y factor de condición-peso (Spearman, $\mathrm{r}_{\mathrm{s}}=0.45$, $\mathrm{P}=0.0002$ ). Sin embargo, ninguno de estos parámetros biométricos presentó una relación significativa con la frecuencia de EMN.

Incidencia de las estaciones de muestreo en la frecuencia de EMN: Se encontró diferencia significativa (Mann-Whitney, $\mathrm{P}=$ 0.018) en la frecuencia de EMN asociada a la procedencia de los ejemplares de $B$. henni. La frecuencia media de EMN en sangre periférica de los ejemplares capturados en el área de influencia directa de la actividad agroindustrial (estación 2) fue 0.15 (Desviación Estándar, $\mathrm{DE}=0.18$, número de ejemplares, $\mathrm{N}=36$ ). Este valor duplicó la frecuencia de EMN de los peces procedentes de la zona poco alterada (estación 1) cuya media fue $0.06(\mathrm{DE}=0.08, \mathrm{~N}$ =36) (Cuadro 2, Fig. 2).

Incidencia de la época climática en la frecuencia de EMN: En todas las estaciones se encontró la mayor frecuencia de EMN durante la época seca. Para la estación 1, la frecuencia media de EMN durante la época seca fue 0.07 $(\mathrm{DE}=0.09, \mathrm{~N}=18)$ mientras que durante 
CUADRO 2

Datos biométricos y frecuencia de EMN en sangre periférica de peces Brycon henni procedentes de dos estaciones de muestreo del Río Grande

TABLE 2

Biometric data and MNE frequency in peripheral blood of fish Brycon henni from two sampling stations in Río Grande

\begin{tabular}{cccccccc}
$\begin{array}{c}\text { Sitio de } \\
\text { muestreo }\end{array}$ & $\begin{array}{c}\text { Época } \\
\text { climática }\end{array}$ & $\mathrm{N}$ & Longitud & Peso & $\begin{array}{c}\text { Factor de } \\
\text { condición }\end{array}$ & $\begin{array}{c}\text { Frecuencia de } \\
\text { EMN }\end{array}$ & $\begin{array}{c}\text { Frecuencia de EMN } \\
\text { por estación }\end{array}$ \\
Estación 1 & Lluvia 2010 & 18 & $14.56 \pm 2.40$ & $44.06 \pm 29.03$ & $1.13 \pm 0.23$ & $0.05 \pm 0.06$ & $0.06 \pm 0.08^{* c}$ \\
& Seca 2011 & 18 & $16.65 \pm 2.05$ & $61.50 \pm 27.25$ & $1.24 \pm 0.13$ & $0.07 \pm 0.09 * \mathrm{a}$ & $(\mathrm{N}=36)$ \\
Estación 2 & Lluvia 2010 & 18 & $13.52 \pm 2.45$ & $31.94 \pm 29.23$ & $1.08 \pm 0.44$ & $0.04 \pm 0.07 * \mathrm{~b}$ & $0.15 \pm 0.18^{* \mathrm{c}}$ \\
& Seca 2011 & 18 & $14.83 \pm 1.62$ & $40.82 \pm 13.96$ & $1.20 \pm 0.13$ & $0.21 \pm 0.19 * \mathrm{ab}$ & $(\mathrm{N}=36)$ \\
\hline
\end{tabular}

Todos los datos se presentan como media \pm DE. * Diferencia estadísticamente significativa $(\mathrm{P} \leq 0.05)$ entre estaciones de muestreo para la misma época climática (a), entre diferentes épocas climáticas para la misma estación (b) y entre estaciones de muestreo independientemente de la época climática (c).

All data correspond to the mean \pm SD. * Statistically significant difference $(\mathrm{P} \leq 0.05)$ between sampling stations in the same season (a), between different seasons in the same station (b) and between sampling stations independently of the season (c).

la época de lluvias la media fue $0.05(\mathrm{DE}=$ $0.06, \mathrm{~N}=18$ ). Para la estación 2, la frecuencia media de EMN durante la época seca fue 0.21 $(\mathrm{DE}=0.19, \mathrm{~N}=18)$ y presentó diferencia significativa (Mann-Whitney, $\mathrm{P}=0.001$ ) respecto a la media encontrada en la época de lluvias $(0.04 \pm 0.07, \mathrm{~N}=18)$ (Cuadro 2, Fig. 2).

\section{DISCUSION}

Los peces pueden actuar como organismos centinelas para indicar el efecto potencial ante la exposición a agentes genotóxicos de origen químico presentes en el agua, y la aplicación de la prueba de $\mathrm{MN}$ en peces es

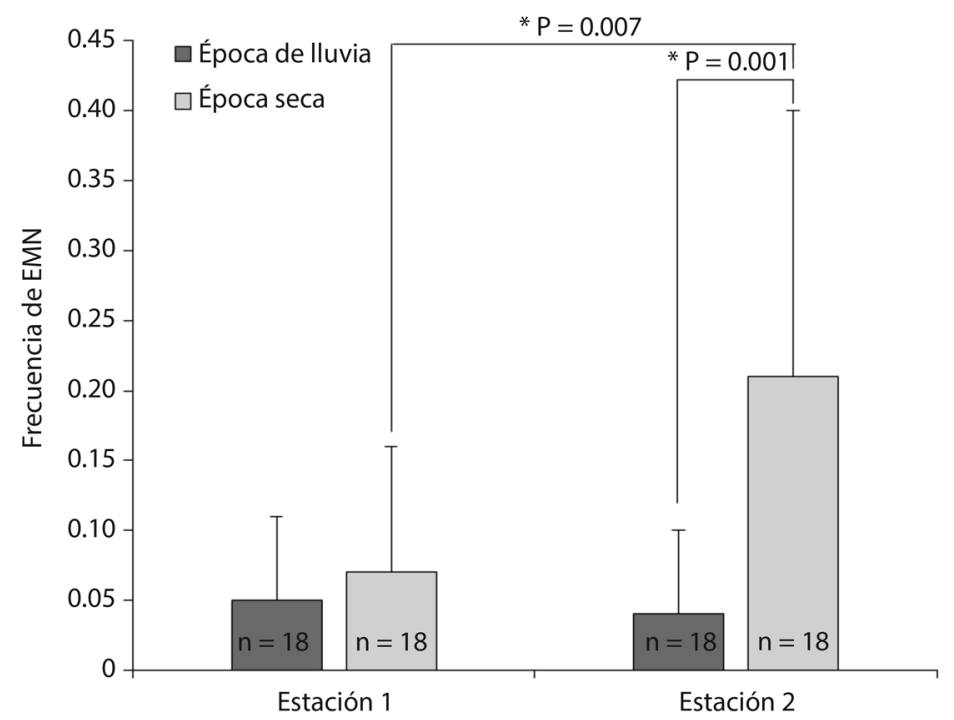

Fig. 2. Frecuencia media de EMN en ejemplares de Brycon henni procedentes de la estación 1 (zona poco alterada) y estación 2 (zona con impacto directo de agroquímicos) en época de lluvia (gris oscuro) y época seca (gris claro). Las barras representan la desviación estándar. El asterisco $(*)$ representa diferencias significativas $(\mathrm{p} \leq 0.05)$.

Fig. 2. Average MNE frequencies in specimens of Brycon henni from station 1 (unimpacted zone) and station 2 (zone with direct agrochemicals impact) in the rainy (dark grey) and dry seasons (light grey). The bars represent the standard deviation. Asterisks $(*)$ represent significant differences $(\mathrm{P} \leq 0.05)$. 
ampliamente utilizada en estos organismos como biomarcador, que permite la detección temprana de problemas ambientales en los ecosistemas acuáticos (Al-Sabti \& Metcalfe, 1995; Çavaş \& Ergene-Gözükara, 2005; Lopez-Barea, 1996; Murthy et al., 2013; Nwani et al., 2010, 2011; Udroiu, 2006). Los peces de la especie Carassius auratus han demostrado un incremento en la frecuencia de EMN como respuesta a la exposición a pesticidas y herbicidas ampliamente usados en agricultura (Cavas, 2011; Çavaş \& Könen, 2007). En la especie Carassius carassius se describió incremento en la cantidad de quiebres en el ADN de especímenes capturados en aguas afectadas por actividades agrícolas en comparación con ejemplares procedentes de una zona sin ese tipo de descargas (Polard et al., 2011a).

Hurtado-Alarcón et al. (2007) reportaron a $B$. henni como una especie con potencial como bioindicadora de la calidad del ambiente al mostrar sensibilidad ante la presencia de agentes genotóxicos en el agua mediante el uso del test de MN (Hurtado-Alarcón et al., 2007). Los resultados del presente estudio confirman dicho potencial al reportar un valor considerablemente superior en la frecuencia de EMN de los ejemplares de $B$. henni procedentes de una zona del Río Grande con influencia directa de la zona bananera que experimenta descargas de agroquímicos con potencial genotóxico y mutagénico.

La frecuencia de EMN significativamente mayor en la sangre de individuos capturados en la época seca, podría indicar aumento de las concentraciones de los xenobióticos como resultado de la reducción de los caudales y de la velocidad del flujo de agua. Durante la aplicación de los plaguicidas, las lluvias de corta duración durante la época seca facilitan el transporte de residuos a las aguas superficiales (Ferenczi, Ambrus, Wauchope, \& Sumner 2002, Polard et al. 2011b, Schulz, 2001). En las corrientes de agua dulce con alta contaminación, está descrito el incremento diez veces superior en la concentración de algunos herbicidas y fertilizantes y en la frecuencia de EMN en peces luego de su aplicación en la época seca (Taghavi et al., 2010, Polard et al., 2011a).

En la zona de estudio se usan permanentemente agroquímicos como fertilizantes y herbicidas relacionados con la agricultura de plátano y banano, principal actividad económica de la región. Esos compuestos ingresan inicialmente a las plantaciones $\mathrm{y}$, debido a que los canales de riego están en contacto directo con los ríos, terminan finalmente llegando a los causes principales como el Río Grande en Turbo - Antioquia. Entre los principales mecanismos de acción asociados a la exposición a agroquímicos se encuentran daños en genes, proteínas, alteraciones enzimáticas, cambios en la replicación del ADN y alteraciones que llevan a mutaciones (Çavaş \& Könen, 2007; Murthy et al., 2013; Ullah \& Zorriehzahra, 2015). Estos últimos mecanismos están implicados en los efectos clastogénicos observados en eritrocitos de peces, como $B$. henni, expuestos a estos agentes.

Los resultados de este estudio confirman la importancia de la cuantificación de la frecuencia de EMN en sangre periférica de peces como un marcador biológico eficiente de la presencia de contaminantes genotóxicos en el ambiente, para programas de monitoreo y control de la calidad del agua en áreas afectas por el desarrollo de actividades económicas que pueden afectar la calidad del aguas. Además, se confirma la sensibilidad de la sabaleta (B. henni) ante la presencia de genotóxicos en el ambiente y su uso como organismo centinela en estudios de monitoreo ambiental como indicadora de la calidad del agua.

\section{AGRADECIMIENTOS}

Agradecemos la financiación de esta investigación al Fondo para el Desarrollo de la Investigación en las Regiones de la Dirección de Regionalización y la Vicerrectoría de Investigación a través del Comité para el Desarrollo de la Investigación -CODI- 2009. 


\section{RESUMEN}

La producción agrícola de monocultivo permanente de banano y plátano en la parte media del Río Grande (Turbo - Antioquia) requiere de la aplicación de diferentes plaguicidas. Prácticas inapropiadas en la producción y cultivos de plátano realizadas en esta región a menudo conllevan a contaminación con agroquímicos que llegan a este cuerpo de agua por procesos de lixiviación y escorrentía. Los peces son los vertebrados más utilizados como especies bioindicadoras de la calidad del agua porque son muy sensibles a la presencia de contaminantes en el ambiente. El objetivo de esta investigación fue comparar la frecuencia de eritrocitos micronucleados $(\mathrm{EMN})$ en sangre periférica de ejemplares de peces sabaleta (Brycon henni) procedentes de dos estaciones del Río Grande que difieren en su grado de contaminación por agroquímicos. Se evaluó la frecuencia de EMN en sangre periférica de peces $B$. henni procedentes de cada estación durante dos épocas de lluvia de 2010 y dos época secas de 2011. Las muestras de sangre fueron obtenidas de la vena caudal, fijadas durante 24 horas y luego teñidas con Giemsa. La frecuencia de EMN fue significativamente mayor en el área impactada por agroquímicos. La frecuencia media de EMN fue mayor en la estación con impacto directo de agroquímicos $(0.15 \pm 0.18)$ que en la estación poco alterada $(0.06 \pm 0.08)$. Además, la frecuencia de EMN en $B$. henni fue significativamente mayor en la época seca. Los resultados de este estudio indican que el análisis de las EMN en B. henni puede ser recomendada como un método adecuado para la detección in situ de agentes genotóxicos en el ambiente.

Palabras clave: frecuencia de eritrocitos micronucleados, biomarcadores, agroquímicos, Brycon henni, daño en el ADN.

\section{REFERENCIAS}

Akpoilih, B. U. 2012. Fish ecogenotoxicology: an emerging science, an emerging tool for environmental monitoring and risk assessment. Global Journal of Bioscience and Biotechnology, 1(2), 141-151.

Al-Sabti, K. (1985). Frequency of chromosomal aberrations in the rainbow trout (Salmo gairdini Rich) exposed to live pollutants. Journal of Fish Biology, 26(1), 13-19.

Al-Sabti, K., \& Metcalfe, C. D. (1995). Fish micronuclei for assessing genotoxicity in water. Mutation Research/Genetic Toxicology, 343(2), 121-135.

Ali, D., Nagpure, N. S., Kimar, S., Kumar, R., \& Kushwaha, B. (2008). Genotoxicity assessment of acute exposure of chlorpyriphos to fresh water fish Channa punctatus (Bloch) using micronucleus assay and alkaline single cell gel electrophoresis. Chemosphere, 71, 1823-1831.
Anderson, S. L., \& Wild, G. C. (1994). Linking genotoxic responses and reproductive success in ecotoxicology. Environmental Health Perspectives, 102 (Suppl. 12), 9-12.

Bonilla-Arboleda, J. P., Peinado, J. E., Urdaneta, M. A., \& Carrascal, E. (2000). Reducción del escurrimiento de plaguicidas al mar caribe. Informe Nacional sobre el uso y manejo de plaguicidas en Colombia, tendiente a identificar y proponer alternativas para reducir el escurrimiento de plaguicidas al mar Caribe. Bogotá, Colombia: Proyecto GRF PDF-B. Global Environment Facility, Ministerio del Medio Ambiente.

Cavas, T. (2011). In vivo genotoxicity evaluation of atrazine and atrazine-based herbicide on fish Carassius auratus using the micronucleus test and the comet assay. Food and Chemical Toxicology, 49(6), 1431-1435.

Çavaş, T., \& Ergene-Gözükara, S. (2005). Micronucleus test in fish cells: a bioassay for in situ monitoring of genotoxic pollution in the marine environment. Environmental and molecular mutagenesis, 46(1), 64-70.

Çavaş, T., \& Könen, S. (2007). Detection of cytogenetic and DNA damage in peripheral erythrocytes of goldfish (Carassius auratus) exposed to a glyphosate formulation using the micronucleus test and the comet assay. Mutagenesis, 22(4), 263-268.

CORPOURABA (Corporación para el Desarrollo Sostenible del Urabá). 2007. Establecimiento de los objetivos de calidad, requerimiento de los planes de saneamiento y manejo de vertimientos (PSMV) a las entidades prestadoras del servicio de alcantarillado de la jurisdicción de Corpourabá. Cuencas de los ríos Turbo, Currulao, río Grande y otras corrientes receptoras de vertimientos del municipio de Turbo. Unidad de Aguas, Subdirección Gestión y Administración Ambiental.

Dahl, G. (1971). Los Peces del Norte de Colombia. Bogotá, Colombia: Ministerio de Agricultura, Instituto de Desarrollo de los Recursos Naturales Renovables INDERENA.

De Flora, S., Bagnasco, M., \& Zanacchi, P. (1991). Genotoxic, carcinogenic, and teratogenic hazards in the marine environment, with special reference to the Mediterranean Sea. Mutation Research/Reviews in Genetic Toxicology, 258(3), 285-320.

De Flora, S., Vigano, L., D’Agostini, F., Camairano, A., Bagnasco, M., Bennicelli, C., Melodia, F., \& Arillo, A. (1993). Multiple genotoxicity biomarkers in fish exposed in situ to polluted river water. Mutation Research/Genetic Toxicology, 319(3), 167-177.

Ergene, S., Çavaş, T., Çelik, A., Köleli, N., Kaya, F., \& Karahan, A. (2007). Monitoring of nuclear abnormalities in peripheral erythrocytes of three fish species from the Goksu Delta (Turkey): genotoxic damage in relation to water pollution. Ecotoxicology, 16(4), 385-391. 
Fenech, M. (2000). The in vitro micronucleus technique. Mutation Research/Fundamental and Molecular Mechanisms of Mutagenesis, 455(1-2), 81-95.

Ferenczi, J., Ambrus, Á., Wauchope, R. D., \& Sumner, H. R. (2002). Persistence and runoff losses of 3 herbicides and chlorpyrifos from a corn field in the Lake Balaton watershed of Hungary. Journal of Environmental Science and Health, Part B, 37(3), 211-224.

Giesy, J. P., Dobson, S., \& Solomon, K. R. (2000). Ecotoxicological risk assessment for Roundup ${ }^{\circledR}$ herbicide. Reviews of Environmental Contamination and Toxicology, 167, 35-120.

Goksøyr, A. (1991). A semi-quantitative cytochrome P450IA1 ELISA: a simple method for studying the monooxygenase induction response in environmental monitoring and ecotoxicological testing of fish. Science of the Total Environment, 101(3), 255-262.

Gollapudi, B. B., Mendrala, A. L., \& Linscombe, V. A. (1995). Evaluation of the genetic toxicity of the organophosphate insecticide chlorpyrifos. Mutation Research/Genetic Toxicology, 342(1), 25-36.

Grisolia, C. K., \& Starling, F. L. (2001). Micronuclei monitoring of fishes from Lake Paranoá, under influence of sewage treatment plant discharges. Mutation Research/Genetic Toxicology and Environmental Mutagenesis, 491(1), 39-44.

Hurtado-Alarcón, J. C., Solarte-David, V. A., López-Ortiz, J. B., \& Montoya-Herrera, F. L. (2007). Prueba de micronúcleos en eritrocitos de sabaletas (Brycon henni E.) presentes en el río Porce y en el Embalse Porce II, Antioquia. Revista Electrónica de Ingeniería en Producción Acuícola, 12, 238-245.

INVEMAR (Instituto de Investigaciones Marinas y Costeras "José Benito Vives de Andréis"). 2004. Diagnóstico y evaluación de la calidad ambiental marina en el Caribe y Pacífico Colombiano. Red de vigilancia para la conservación y protección de las aguas marinas y costeras de Colombia; Diagnóstico nacional y regional 2004 (Informe final, 298 p.). Santa Marta.

Jha, A. N. (2004). Genotoxicological studies in aquatic organisms: an overview. Mutation Research/Fundamental and Molecular Mechanisms of Mutagenesis, 552(1), 1-17.

Lakra, W. S., \& Nagpure, N. S. (2009). Genotoxicological studies in fish: a review. Indian Journal of Animal Sciences, 79, 93-98.

Lopez-Barea, J. (1996). Biomarkers to detect environmental pollution. Toxicology Letters, 88, 77-79.

Mintrabajo (Ministerio de Trabajo República de Colombia). (2013). Estudio de Perfil Productivo Rural del Municipio de Turbo. Ministerio de Trabajo - Programa de las Naciones Unidas para el Desarrollo.
Fuente: http://www.mintrabajo.gov.co/component/ docman/doc_download/1634-perfilturbo.html

Mojica, J. I., Castellanos, C., Usma, J. S., \& Álvarez, R. (Eds.) (2002). Libro rojo de peces dulceacuícolas de Colombia. La serie libros rojos de especies amenazadas de Colombia. Bogotá: Instituto de Ciencias naturales, Universidad Nacional de Colombia, Ministerio del Medio Ambiente.

Montoya-López, A. F., Carrillo, L. M., \& Olivera-Ángel, M. (2006a). Algunos aspectos biológicos y del manejo en cautiverio de la Sabaleta Brycon henni Eigenmann, 1913 (Pisces: Characidae). Revista Colombiana de Ciencias Pecuarias, 19(2), 180-186.

Montoya-López, A. F., Tabares, C. J., Echeverri, A., Arboleda, L., \& Olivera-Ángel, M. (2006b). Descripción anatómica e histológica de las gónadas en Sabaleta (Brycon henni, Eigenmann 1913). Revista Colombiana de Ciencias Pecuarias, 19(2), 187-196.

Murthy, K. S., Kiran, B. R., \& Venkateshwarlu, M. (2013). A review on toxicity of pesticides in Fish. International Journal of Open Scientific Research, 1(1), 15-36.

Nepomuceno, J. C., Ferrari, I., Spanó, M. A., \& Centeno, A. J. (1997). Detection of micronuclei in peripheral erythrocytes of Cyprinus carpio exposed to metallic mercury. Environmental and Molecular Mutagenesis, 30(3), 293-297.

Nepomuceno, J. C, \& Spanó, M. A. (1992). Incidência de micronúcleos em eritrócitos periféricos de tilápias (Oreochromis mossambicus) provindas de diferentes estações de piscicultura. Revista do Centro de Ciencias Biomédicas da Universidade Federal de Uberlandia, 8(1), 7-16.

Nwani, C. D., Lakra, W. S., Nagpure, R., Kumar, R., Kushwaha, B., \& Srivastava, S. K. (2010). Mutagenic and genotoxic effects of Carbosulphan in freshwater fish Channa punctatus (Bloch) using micronucleus assay and alkaline single-cell gel electrophoresis. Food and Chemical Toxicology, 48(1), 202-208.

Nwani, C. D., Nagpure, N. S., Ravindra, K., Kushwaha, B., Kumar, P., \& Lakra, W. S. (2011). Mutagenic and genotoxic assessment of atrazine - based herbicide to freshwater fish Channa punctatus (Bloch) using micronucleus test and single cell gel electrophoresis. Environmental Toxicology and Pharmacology, 31(2), 314-322.

Obiakor, M. O., Okonkwo, J. C., Nnabude, P. C., \& Ezeonyejiaku, C. D. (2012). Eco-genotoxicology: Micronucleus Assay in Fish Erythrocytes as In situ Aquatic Pollution Biomarker: a Review. Journal of Animal Science Advances, 2(1), 123-133.

Osman, A. G. (2014). Genotoxicity tests and their contributions in aquatic environmental research. Journal of Environmental Protection, 5(14), 1391. 
Polard, T., Jean, S., Gauthier, L., Laplanche, C., Merlina, G., Sánchez-Pérez, J. M., \& Pinelli, E. (2011a). Mutagenic impact on fish of runoff events in agricultural areas in south-west France. Aquatic Toxicology, 101(1), 126-134.

Polard, T., Jean, S., Merlina, G., Laplanche, C., Pinelli, E., \& Gauthier, L. (2011b). Giemsa versus acridine orange staining in the fish micronucleus assay and validation for use in water quality monitoring. Ecotoxicology and Environmental Safety, 74(1), 144-149.

Rahman, M. F., Mahboob, M., Danadevi, K., Banu, B. S., \& Grover, P. (2002). Assessment of genotoxic effects of chloropyriphos and acephate by the comet assay in mice leucocytes. Mutation Research/ Genetic Toxicology and Environmental Mutagenesis, 516(1), 139-147.

Ricker, W. (1975). Computation and interpretation of biological statistics of fish populations. Bulletin of the fisheries research board of Canada, 191, 382.

Rubio, M. \& Silveira, R. (2009). Bienestar de los animales acuáticos, con fines de control sanitario. Revista Electrónica de Veterinaria [Internet], 10, 1-12.

Ruiz, M. J., \& Marzin, D. (1997). Genotoxicity of six pesticides by Salmonella mutagenicity test and SOS chromotest. Mutation Research/Genetic Toxicology and Environmental Mutagenesis, 390(3), 245-255.

Schulz, R. (2001). Rainfall-induced sediment and pesticide input from orchards into the Lourens River, Western Cape, South Africa: importance of a single event. Water Research, 35(8), 1869-1876.

Shugart, L., \& Theodorakis, C. (1998). New trends in biological monitoring: application of biomarkers to genetic ecotoxicology. Biotherapy, 11(2), 119-127.
Taghavi, L., Probst, J. L., Merlina, G., Marchand, A. L., Durbe, G., \& Probst, A. (2010). Flood event impact on pesticide transfer in a small agricultural catchment (Montousse at Aurade, south west France). International Journal of Environmental and Analytical Chemistry, 90(3-6), 390-405.

Talapatra, S. N., Ganguly, P., Mukhopadhyay, A., \& Banerjee, S. K. (2007). Assessment of genetic biomarkers with special reference to micronucleated and binucleated erythrocytes in two fish species grown at Industrial vicinity of thermal power plants, Kolkata, India. Asian Journal of Water, Environment and Pollution, 4(1), 139-144.

Udroiu, I. (2006). The micronucleus test in piscine erythrocytes. Aquatic Toxicology, 79(2), 201-204.

UICN (Unión Internacional para la conservación de la naturaleza). (2003). Lista roja de especies en peligro. URL: http://www.redlist.org

Ullah, S., \& Zorriehzahra, M. J. (2015). Ecotoxicology: a review of pesticides induced toxicity in fish. Advances in Animal and Veterinary Sciences, 3(1), 40-57.

Vivas-Aguas, L. J., Tosic, M., Sánchez, J., Narvaés, S., Cadavid, B., Bautista, P., Betancour, J., Parra, J., Carvajalino, M., \& Espinosa, L. (2011). Diagnóstico y Evaluación de la calidad ambiental marina en el caribe y pacífico colombiano. Red de vigilancia para la conservación y protección de las aguas marinas y costeras de Colombia-REDCAM. Inf. Téc (Informe técnico). Santa Marta: Invemar.

Zamora, A. P. \& García-Valencia, C. (2007). El golfo: un espacio socioeconómico. Atlas del golfo de Urabá: una mirada al Caribe de Antioquia y Chocó (pp. 129149). Instituto de Investigaciones Marinas y Costeras -INVEMAR- y Gobernación de Antioquia. 